\title{
Dietary intake of whole grain in Irish adults
}

\author{
A. M. Burns, N. F. C. Devlin, B. A. McNulty, M. J. Gibney and A. P. Nugent \\ UCD Institute of Food and Health, University College Dublin, Belfield, Dublin 4, Ireland.
}

Limited data exists on dietary intakes of whole grain in an Irish population. The objective of the present study was to characterise whole grain consumption in a representative sample of Irish adults, using data from the National Adult Nutrition Survey (NANS). NANS collected detailed information on habitual food and beverage consumption, using a 4 day food diary, in 1500 Irish adults from $2008-2010^{(1)}$. Whole grain foods were identifiedand information on the grain ingredients was ascribed from food packaging information and by contacting manufacturers ${ }^{(2)}$. After the exclusion of under reporters, dietary intakes of whole grain were characterised and compliance with dietary recommendations assessed ${ }^{(3)}$.

\begin{tabular}{|c|c|c|c|c|c|c|c|}
\hline & \multicolumn{3}{|c|}{ Total Population } & \multicolumn{4}{|c|}{ Consumers only } \\
\hline & $n$ & Mean & SD & $n$ & $\%$ & Mean & SD \\
\hline $\begin{array}{l}\text { Total Population } \\
\text { Sex }\end{array}$ & 1051 & 29.4 & 37.0 & 838 & 79.7 & 36.9 & 37.9 \\
\hline Male & 523 & $33.3^{\mathrm{a}}$ & 44.7 & 411 & 78.6 & $42.4^{\mathrm{a}}$ & 46.5 \\
\hline Female & 528 & $25.6^{\mathrm{b}}$ & 26.6 & 427 & 80.9 & $31.7^{\mathrm{b}}$ & 26.2 \\
\hline \multicolumn{8}{|l|}{ Age } \\
\hline $18-35$ years & 377 & $32.5^{\mathrm{ns}}$ & 45.7 & 300 & 79.6 & $40.9^{\mathrm{ns}}$ & 47.8 \\
\hline $35-50$ years & 308 & 29.0 & 31.1 & 254 & 82.5 & 35.2 & 31.0 \\
\hline $50-65$ years & 204 & 26.6 & 30.1 & 164 & 80.4 & 33.1 & 30.2 \\
\hline$\geqslant 65$ years & 162 & 26.7 & 31.5 & 120 & 74.1 & 36.0 & 31.7 \\
\hline \multicolumn{8}{|l|}{ Social Class } \\
\hline Professional/managerial & 484 & $31.1^{\mathrm{ns}}$ & 34.7 & 403 & 83.3 & $37.3^{\text {ns }}$ & 34.9 \\
\hline Non-manual & 183 & 29.1 & 34.5 & 145 & 79.2 & 36.7 & 35.0 \\
\hline Skilled manual & 142 & 25.3 & 29.4 & 110 & 77.5 & 32.6 & 29.5 \\
\hline Semi-skilled/unskilled & 205 & 29.5 & 48.1 & 154 & 75.1 & 39.3 & 52.0 \\
\hline \multicolumn{8}{|l|}{ BMI Measured } \\
\hline \% Underweight & 6 & 44.8 & 55.9 & 4 & 66.7 & 67.2 & 56.5 \\
\hline$\%$ Normal weight & 387 & 28.8 & 38.2 & 311 & 80.4 & 35.8 & 39.6 \\
\hline$\%$ Overweight & 359 & $35.5^{\mathrm{a}}$ & 38.6 & 305 & 85.0 & 41.7 & 38.6 \\
\hline$\%$ Obese & 153 & $25.0^{\mathrm{b}}$ & 29.8 & 121 & 79.1 & 31.7 & 30.3 \\
\hline \multicolumn{8}{|l|}{ Waist-to-hip ratio } \\
\hline Increased risk & 417 & $34.1^{\mathrm{a}}$ & 42.5 & 347 & 41.4 & $41.0^{\mathrm{a}}$ & 43.5 \\
\hline High risk & 439 & $27.2^{\mathrm{b}}$ & 29.7 & 355 & 42.4 & $33.6^{\mathrm{b}}$ & 29.6 \\
\hline
\end{tabular}

a,b Different uppercase superscript letters indicate mean differences within each factor using Independent $t$-test or One way ANOVA with Bonferroni correction $P<0.05$.

Mean intakes (g/d) were 29.4 rising to 36.9 for consumers only, with $79.7 \%$ of the Irish population consumers of whole grain. Breakfast and the home were the main contributing meal and location for whole grain consumption, with mean intakes of 15.8 and $26.7 \mathrm{~g} / \mathrm{d}$ respectively. 'Breads and rolls' was the major contributor to whole grain intakes (40.9\%) followed by 'ready to eat breakfast cereals' (33\%). Wheat was the major grain type consumed (68\%), with oats also contributing considerably (19\%). Only $21 \%$ of the population achieved the recommended intakes of $48 \mathrm{~g} / \mathrm{d}^{3}$. This study indicates that although whole grains are widely consumed by Irish adults, actual levels of intake and compliance with dietary recommendations are low.

1. National Adult Nutrition Survey Summary Report (2011) Available at: http://www.iuna.net (Accessed at 04/03/2013).

2. Devlin NFC, McNulty BA, Gibney MJ et al. (2012) Br J Nutr 11, 1-9.

3. USDA (2010) Dietary Guideines for Americans. www.dietaryguidelines.gov.(Accessed at 04/03/2013). 